P7 WHAT ARE THE RISKS OF ALLERGY PREVENTION? A SERIES OF THREE CASES OF SESAME ANAPHYLAXIS ON FIRST INGESTION

Emma Ruth*, Aideen Byrne. Our Lady's Children's Hospital, Crumlin, Dublin, Ireland

10.1136/archdischild-2019-epa.363

Background The prevalence of food allergy amongst young children in the developed world is estimated to be $5 \%$. Recently published data demonstrates that early introduction of food allergens prevents food allergy ${ }^{1}$. There remains much controversy over whether these foods should be introduced with or without prior allergy skin testing. On one hand, access to such testing, is universally delayed due to limited resources. On the other hand, introduction without testing may trigger an allergic reaction. New UK and Australian guidance have advised introduction of peanut and egg at 6 months without testing. To date, no life-threatening reactions have been reported related to the introduction of these allergenic foods.

However, it remains unclear whether other major allergens such as sesame, could be introduced in this way. Sesame allergy affects approx. $0.1 \%$ of the population but is more prevalent in areas with high consumption such as the Middle East where it is the second commonest cause of anaphylaxis in Israeli children ${ }^{2}$.

The aim of this cases series is to highlight three cases of sesame anaphylaxis in infants under one year. The sesame was introduced at home without prior testing.

Methods A retrospective chart review was undertaken of known cases of sesame anaphylaxis in infants, admitted to our tertiary Paediatric Hospital.

Results Our patients ranged in age from 6 to 8 months and all presented via the Emergency Department. They all received tahini-containing hummus in the home setting for the first time prior to presentation. Patient one developed perioral erythema, facial flushing, angioedema, tongue swelling and profuse vomiting, he was administered adrenaline by ambulance personnel. Patient two became immediately distressed, developed stridor with facial and lip swelling, vomiting and diarrhea. He received two doses of adrenaline pre-hospital. Patient three developed immediate perioral urticaria spreading to his neck. He became pale and lethargic and vomited. This patient did not receive adrenaline but was managed with antihistamines and oral corticosteroids.

All three infants showed subsequent positive SPT to sesame (ranging from 4-6 mm) with negative testing for chickpea.

Conclusion Early introduction of peanut is now taking place worldwide with no reported cases to date of anaphylaxis. Here we present 3 cases of sesame anaphylaxis from just one institution. This case series highlights that early introduction of food allergens is likely to have differential risk profiles, with sesame seed introduction likely to carry a higher risk if introduced to all infants without prior screening.

\section{P8 FOLLOW UP OF SUBLINGUAL IMMUNOTHERAPY IN A PAEDIATRIC POPULATION AT CORK UNIVERSITY HOSPITAL}

Sinead O'Donnell ${ }^{*}$, Megan Morris, Jonathan Hourihane. Department of Paediatrics, Cork University Hospital, Cork, Ireland

10.1136/archdischild-2019-epa.364
Introduction Allergic rhinitis is an immune-mediated response of the nasal mucosa to allergens, which is clinically characterised by paroxysms of sneezing, rhinnorhea, and nasal obstruction which is often accompanied by itching of the eyes, nose and palate. In the most severe cases, patients can still have a reduced quality of life despite optimal treatment with antihistamines, eye drops and nasal sprays. When the symptoms of allergic rhinitis persist, they can have a dramatic impact on the quality of life of the child and their family. Untreated symptoms can have a detrimental effect the child's sleeping pattern which consequently can impact negatively on their growth potential, cognition and participation in social activities. Sublingual Immunotherapy (SLIT) can alter a patient's sensitivity to inhaled allergens and improve symptoms which do not respond to the standard therapies.

Aim and methods The aims of this study were to assess the effect SLIT had on patients both during the treatment and after. Effects were determined by retrospective chart review. 38 children (age 5-17) completed 3 years of SLIT at Cork University Hospital between 2010 and 2016. 34 patient/guardians were contacted by telephone to provide additional information; the remaining 4 were unreachable by phone.

Results In the study population, Immunotherapy was prescribed for house dust mites (24\%) and grass pollen (76\%). Treatment with SLIT reduced symptoms of allergic rhinitis in $91 \%$ of patients during SLIT. $62 \%$ had an improvement in symptoms post completion of therapy and $29.4 \%$ maintained the level of reduction during treatment. There was a reported drop in the amount of patients using medications such as antihistamines, nasal sprays and eye drops. $50 \%$ of the patients had a diagnosis of asthma as well as allergic rhinitis. $76 \%$ of these patients had an improvement of their asthma symptoms, resulting in a reduction of inhaler use. Overall, $88 \%$ of the patients were satisfied with the SLIT and they would recommend it to others.

Discussion The effect of grass pollen and HDM allergen SLIT for allergic rhinitis is substantial and sustained in medium term, patients experienced a reduction in both symptoms and medication use. There is also an effect of SLIT on asthma which must be noted, as these patients also experienced a reduction in symptoms and medication use.

\section{P9 DOES PENICILLIN ALLERGY EXIST AMONG CHILDREN? A RETROSPECTIVE STUDY ASSESSING THE PREVALENCE OF PENICILLIN ALLERGY AMONG CHILDREN UNDERGOING A PENICILLIN DERIVATIVE CHALLENGE TEST IN THE COMMUNITY, FOLLOWING RASH OCCURRING AFTER TREATMENT WITH A PENICILLIN DERIVATIVE}

${ }^{1}$ Abraham Schechter*, ${ }^{1}$ Menachem Bitan, ${ }^{2}$ Yosef Schechter, ${ }^{1}$ Tatiana Voltsinger. ${ }^{1}$ Meuhedet Health Services, Jerusalem District, Jerusalem, Israel; ${ }^{2}$ Shaarei Zedek Medical Center, Jerusalem, Israel

\subsection{6/archdischild-2019-epa.365}

Background rashes are common among children treated with penicillin derivatives. Many are recorded as 'penicillin allergy' without any testing.

Aim This study aimed to demonstrate that most children are not 'allergic' to penicillin and can therefore use penicillin again in the future. In addition, it aimed to demonstrate that 